\title{
Role of Sex-linked Histocompatibility Antigens
}

Current interest in histocompatibility antigens, apart from technical problems concerning their investigation and practical problems connected with transplantation in man, largely centres on their biological role. As has been frequently pointed out, it is difficult to believe that such a major genetic investment has no biological importance. Differing views on the biological role of the major histocompatibility locus in any species, including HL-A in man and $\mathrm{H}-2$ in mice, have been expressed, ranging from those who see them as connected in some way with the generation of immunoglobulin gene diversity, and those who consider that their function is concerned with the maintenance of the integrity of the individual, being involved in protection either against neoplasms or against the potential invasive problems which accompany the evolution of viviparity. These views have recently been discussed in the pages of Nature by Bodmer $(237,139 ; 1972)$ and Burnet (245, 359; 1973).

It is also legitimate to ask, however, whether the 'minor' histocompatibility loci, and indeed other antigenic polymorphisms such as the ABO blood group system in man, also play a part in the functions suggested for the major histocompatibility locus. Some of the minor loci in the mouse, the only species studied in any detail, seem to be almost as complex as $\mathrm{H}-2$, and once again it would be good to think that such a major genetic investment supposes some biological function. Is this function a modification or elaboration of the functions of the major locus, or do the minor loci have some distinct part to play?

If such questions become puzzling for the minor autosomal loci, they become positively bewildering when related to $\mathrm{X}$ and $\mathrm{Y}$-linked histocompatibility antigens. Whatever the significance of histocompatibility loci in general, $\mathrm{X}$ and $\mathrm{Y}$-linked $\mathrm{H}$ loci are clearly a very special case, and many of the possible functions of autosomal loci clearly cannot be carried out by sex-linked loci. There is much less hard information about the $\mathrm{X}$ and $\mathrm{Y}$ linked antigens, partly because of the technical problems of their investigation. Recently considerable advances in knowledge of one such system, the H-Y antigen in the mouse, have been made, stemming from the technical advances in detection of $\mathrm{H}-\mathrm{Y}$ antigens made by Boyse and his colleagues.

The results of one such study by Bennett and Boyse are reported on page 308 of this issue of Nature. They reinvestigated the question of whether immunisation against $\mathrm{H}-\mathrm{Y}$ antigens in female mice might alter the sex ratio in their progeny. They remind readers that such investigations have been conducted before, but always by attempting to immunise the female in vivo, and that such investigations have always been negative or equivocal, but that it was previously impossible to decide which of the many possible reasons for this failure to demonstrate immunoselection was correct. They exposed sperm to anti-H-Y and complement in vitro, and then artificially inseminated females, and did indeed show a small but statistically significant decrease in the proportion of male offspring in litters of such females.
Bennett and Boyse discuss, in an admirably cautious way, the conclusions to be drawn from their observations. Two points may be emphasised. One is that this is some evidence that the H-Y gene may be concerned with the sex ratio, a conclusion which one would hesitate to make a priori when one considers the ragbag of genes known to be present on the sex chromosomes. The other point that should be stressed is that although the alteration in the sex ratio observed is small, and complete elimination of male offspring was not achieved, the alteration in the sex ratio was from $53 \%$ males in untreated litters to $45 \%$ in experimental litters, that is, an increase of $8 \%$ in the proportion of females. Although small, one can imagine many situations in farm livestock where such a change, if accompanied by no decrease in fertility, would be of considerable economic importance.

From a Correspondent

\section{Hot Spots Must Move}

If linear chains of volcanoes in the ocean basins originate as outflows from localised regions near the Earth's surface-hot spots-over which plates have moved during geological times, then these hot spots cannot have formed a fixed frame of reference during the past 40 million years. This is the conclusion of Molnar and Atwater in this issue of Nature (page 288). This result has major implications for the hot spot hypothesis as there has up to now been a hope among geophysicists that hot spots might have been semi-permanent features and thus good markers of absolute motion of plates over the mantle. The implications, however, go beyond the geometry. If hot spots are not stationary relative to each other, there are serious questions to be asked about their physical character and how it permits them to migrate.

Since the idea of very active volcanic sources leaving their trace on plates was revived by Morgan three years ago, many groups have attempted to use the concept to learn more about past motions. The results have been strangely inconclusive. The one undeniable thing from the geological and geophysical evidence is that the long traces ending in present day volcanoes do indicate something other than pure chance. Many geophysicists would further agree that there is something global to the traces- they are not ten or so entirely unrelated phenomena. But beyond there, opinions differ. Are hot spots simply localised lava sources, or do they have any vertical extension? Are the traces markers of pre-existing fractures or can hot spots punch volcanoes in unfractured plate? Present day geometry of the traces, palaeomagnetic data and a reasonable understanding of plate positions for the past 70 million years are all one has to go by at the moment.

Hargraves and Duncan (Nature, 245, 361; 1973) analysed palaeomagnetic data and concluded that they were inconsistent with hot spots being stationary relative 